\title{
IgG1 responses following SARS-CoV-2 infection are polyclonal and highly personalized, whereby each donor and each clone displays a distinct pattern of cross-reactivity against SARS-CoV-2 variants
}

Danique M.H. van Rijswijck ${ }^{1,2, \ddagger}$, Albert Bondt ${ }^{1,2, \ddagger}$, Max Hoek ${ }^{1,2}$, Karlijn van der Straten ${ }^{3,4}$, Tom G. Caniels $^{3}$, Meliawati Poniman ${ }^{3}$, Dirk Eggink ${ }^{5}$, Chantal Reusken ${ }^{5}$, Godelieve J. de Bree ${ }^{4}$, Rogier W. Sanders $^{3}$, Marit J. van Gils ${ }^{3}$, Albert J.R.Heck ${ }^{1,2, *}$

${ }^{1}$ Biomolecular Mass Spectrometry and Proteomics, Bijvoet Center for Biomolecular Research and Utrecht Institute for Pharmaceutical Sciences, University of Utrecht, Padualaan 8, Utrecht $3584 \mathrm{CH}$, the Netherlands

${ }^{2}$ Netherlands Proteomic Center, Padualaan 8, Utrecht $3584 \mathrm{CH}$, the Netherlands

${ }^{3}$ Department of Medical Microbiology, Amsterdam UMC, University of Amsterdam, Amsterdam Institute for Infection and Immunity, Meibergdreef 9, Amsterdam 1105 AZ, the Netherlands.

${ }^{4}$ Department of Internal Medicine, Amsterdam UMC, Vrije Universiteit Amsterdam, Amsterdam Institute for Infection and Immunity, Meibergdreef 9, Amsterdam 1105 AZ, the Netherlands.

${ }^{5}$ National Institute for Public Health and the Environment, RIVM, Antonie van Leeuwenhoeklaan 9, 3721 MA Bilthoven, the Netherlands

¥These authors contributed equally

*Corresponding Author: Albert J.R. Heck, E-mail: a.j.r.heck@uu.nl

\section{Abstract}

Using a recently introduced efficient mass spectrometry-based approach we monitored in molecular detail the IgG1 clonal responses in individual donorsindividual donors' IgG1 clonal responses in molecular detail, examining SARSCoV-2 spike-protein-specific IgG1 repertoires. We monitored the plasma clonal IgG1 profiles of 8 donors (4 male and 4 female) who had recently experienced an infection by either the wild type Wuhan Hu-1 virus or one of 3 VOCs (Alpha, Beta and Gamma). In these donors we charted the full plasma IgG1 repertoires as well as the IgG1 repertoires targeting the SARS-CoV-2 spike protein trimer as antigen. We observed that shortly after infection in between $<0.1 \%$ to almost $10 \%$ of all IgG1 antibody molecules present in plasma did bind to the spike protein. Each donor displayed a unique plasma IgG1 repertoire, but also each donor displayed a unique and polyclonal antibody response against the SARS-CoV-2 spike-protein variants. Our analyses revealed that certain clones exhibit (alike) binding affinity towards all four tested spike-protein variants, whereas other clones displayed strong unique mutant-specific affinity. We conclude that each infected person generates a unique polyclonal response following infection, whereby some of these clones can bind multiple viral variants, whereas other clones do not display such cross-reactivity. In general, by assessing IgG1 repertoires following infection it becomes possible to identify and select fully matured human plasma antibodies that target specific antigens, and display either high specificity or cross-reactivity versus mutated versions of the antigen, which will aid in selecting antibodies that may be developed into biotherapeutics. 


\section{Introduction}

Since the outbreak, the SARS-CoV-2 virus has spread rapidly around the world and is continuously presenting itself in new variants (1). While most mutations are mildly deleterious, certain mutations lead to variants with altered virus characteristics, affecting transmissibility and antigenicity (1). Variants that affect virus characteristics and that cause significant community transmission have been declared Variants of Concern (VOC) (2-4). Currently, five mutated SARS-CoV-2 VOCs have been annotated by the WHO: Alpha (B.1.1.7), Beta (B.1.351), Gamma (B.1.1.28.P1), Delta (B.1.617.2) and Omicron (B.1.1.529), although the latter seems to be leading to less severe disease (2). Since the virus has spread globally, and also due to intense worldwide vaccination programs, a substantial amount of the population has created an immune response and subsequent memory towards the virus (5).

However, with the emergence of new VOCs, the question becomes relevant whether the humoral immunity that is gained after infection with one VOC also provides protection against another VOC. This cross-reactive protection against different variants would be essential to ultimately combat the virus. Additionally, it may be interesting to see whether there are differences in response and cross-reactivity between individuals, in between different antigen directed Ig clones, and in between SARS-CoV-2 variants. Previous reports on this topic have suggested that new variants can (partially) escape humoral immune responses, since no clear binding or neutralization was observed in vitro (4). However, in vitro assays do not provide the full picture of the immune response and in vivo studies are needed to support this further. Moreover, the currently available assays used to assess cross-reactivity against different VOCs, target the response at the level of the total antibody pool, overlooking the fact that our immune system provides a polyclonal response creating multiple different antibody clones against a single antigen. In principle, all these different clones would give a unique response and can therefore show broad variation in the degree of cross-reactivity towards the antigens of other VOCs. Recently, there have been advances in techniques for antibody repertoire profiling, such as next-generation sequencing (NGS) of B cells. However, this technique focuses on the circulating $B$ cell population which might not represent the antibodies in circulation. Furthermore, it does not provide information regarding the abundances of each clone that will eventually end up in the circulation (6). This defines the unmet need for an approach that provides information on individual antibody clones in the circulation to better understand the elicited antibody response after infection. Subsequently, this information will be crucial for the development of optimal biotherapeutics, that ideally are cross-reactive and neutralizing against all known and/or future VOCs.

Recently, we introduced an approach to monitor qualitatively and quantitatively clonal lgG1 repertoires in plasma, allowing investigation of humoral immunity at the molecular level in detail. In this approach IgGs are purified and subsequently cleaved into the constant domain (Fc) and antigen binding domain (Fab), using an enzyme that cleaves only lgG1s. All released IgG1 Fab molecules, spanning typically a 45 $\mathrm{kDa}<\mathrm{Mw}<53 \mathrm{kDa}$ mass range, are then fractionated and profiled at the intact protein level by liquidchromatography coupled mass spectrometry (LC-MS). As each clone has a distinct mass and retention time (RT), these LC-MS traces provide a qualitative picture of the lgG1 repertoires. By spiking in recombinant lgG1 $\mathrm{mAbs}$ as internal standards each individual plasma clone in the plasma can be quantified (7). In our initial work we focused on total IgG1 repertoires in plasma. Here, we extend this approach, not only monitoring total plasma clonal repertoires, but also we employed recombinant SARS-CoV-2 (2P stabilized) spike trimerproteins (in this manuscript referred to as S-protein) to enrich for the clonal repertoires targeting specifically SARS-CoV-2 S-protein antigens of the studied VOCs. This approach allows us to examine cross-reactivity of 
specific antibody clones against different S-protein variants.

We monitored the SARS-CoV-2 S-protein specific IgG1 polyclonal response in 8 selected donors that had suffered an infection with different SARS-CoV-2 VOCs, in total two donors (one male/one female) per variant. We assessed the antibody binding towards the wild type Wuhan Hu-1 (WT) and three of the VOCs of the SARS-CoV-2 S-protein namely, Alpha, Beta, and Gamma. Our data reveal that the immune system produces in each donor a unique polyclonal lgG1 repertoire against the S-protein. Testing the ability of cross-reactivity of each of the detected clones against other VOCs, we observe a broad spectrum of different responses. Some clones bind equally well to all VOCs S-proteins, whereas others only to one, two or three of the variants.

\section{Material and methods}

\section{Donor characteristics}

The eight donors included were part of the larger cross-sectional COSCA cohort (NL 73281.018.20) as described previously (8). The plasma of these infected adults were collected 3-6 weeks after symptoms onset. All participants had at least one nasopharyngeal or oropharyngeal swab positive for SARS-CoV-2, for half of them (COSCA- 303, 308, 309 and 310) the variant of infection was sequence confirmed. For the other donors the variant of infection was assumed by a proven variant of infection by their household member (COSCA-304 and 307) or because no VOC had emerged yet at the time of sampling (COSCA-002 and 003). Participants were included from the start of the COVID-19 pandemic in the Netherlands in March 2020 until the end of February 2021. The study was conducted at the Amsterdam University Medical Centers, location AMC, in the Netherlands and approved by the local ethical committee of the AMC (NL 73281.018.20). All individuals included in this study gave written informed consent before participating.

\section{Production and Purification of full-length Spike protein trimers of VOCs}

The S-protein antigen constructs representing the different VOCs contained the following mutations compared to the WT variant (Wuhan Hu-1; GenBank: MN908947.3): deletion $(\Delta)$ of $\mathrm{H} 69, \mathrm{~V} 70$ and $\mathrm{Y} 144$, N501Y, A570D, D614G, P681H, T716I, S982A, and D1118H in Alpha (B.1.1.7); L18F, D80A, D215G, L242H, R246I, K417N, E484K, N501Y, D614G, and A701V in Beta (B.1.351); and L18F, T20N, P26S, D138Y, R190S, K417T, E484K, N501Y, D614G, H655Y, and T1027I in Gamma(P.1). The genes were ordered as gBlock gene fragments (Integrated DNA Technologies) and cloned Pst I/Not I in a pPPI4 expression vector containing a hexahistidine (his) tag with Gibson Assembly (Thermo Fisher Scientific). All S constructs were verified by Sanger sequencing and the protein was subsequently produced in human embryonic kidney (HEK) 293F cells (Thermo Fisher Scientific) and purified as previously described $(4,8)$.

\section{SARS-CoV-2 S-protein trimer specific antibody enrichment}

The full-length trimeric S-protein variants of the different VOCs were covalently bound to Pierce NHSActivated Agarose Spin Columns. Four Pierce NHSactivated agarose spin columns, each loaded with a different VOC S-protein variant, were assembled according to manufacturer's instructions, and placed in $2 \mathrm{~mL}$ Eppendorf Tubes. Therefore, each of the spin columns was incubated with $0.5-1.0 \mathrm{mg}$ of one of the variants (either WT, Alpha, Beta or Gamma). The Sproteins together with the beads were then incubated for 2 hours using an end-over-end rotator at room temperature. After incubation with the S-protein, the flowthrough was collected, and the agarose spin columns were washed two times with $400 \mu \mathrm{l}$ PBS. After the washing steps, the agarose spin columns were incubated with $400 \mu \mathrm{l}$ Tris $(1 \mathrm{M}, \mathrm{pH}$ 8) for $30 \mathrm{~min}$ as a quenching buffer, using an end-over-end rotator at room temperature. After incubation with Tris the columns were washed three times with $400 \mu \mathrm{I}$ PBS and subsequently stored at 4 degrees in $300 \mu$ PBS. 
$60 \mu \mathrm{l} \mathrm{S}$-protein-bead slurry was incubated with $200 \mu \mathrm{L}$ of each plasma sample separately (marked as COSCA002, 003. 303, 304, 307, 308, 309, 310) together with $300 \mu \mathrm{l}$ PBS in Pierce spin columns (ThermoFisher Scientific). The different plasma samples were each incubated with the different Sprotein-bead variants for 2 hours (e.g., eight different plasma samples with the four different VOCs SARSCoV-2 S-protein variants) on an end-over-end rotator at room temperature. After incubation with the SARSCoV-2 S-proteins the flowthrough was collected, further referred to as the unbound fraction. Subsequently, the spin columns were washed two times with $600 \mu \mathrm{PBS}$ and two times with $600 \mu \mathrm{l}$ Milli$Q$ water. Hereafter, the SARS-CoV-2 spike specific antibodies could be eluted from the S-proteins-beads. To this end, we added $100 \mu \mathrm{L}$ Glycine- $\mathrm{HCl}(\mathrm{pH} 2.7)$, incubated shaking for $10 \mathrm{~min}$, and collected the socalled bound fraction by centrifugation for $1 \mathrm{~min}$ at 500 $\times g$. This was repeated two more times. The bound fraction was captured in a $1.5 \mathrm{~mL}$ Eppendorf already containing $60 \mu \mathrm{l}$ Tris $(1 \mathrm{M}, \mathrm{pH} 8)$ to be able to directly neutralize the eluted fractions.

\section{Bound, unbound and full plasma IgG purification, and subsequent Fab generation}

For the IgG purification and the generation of Fabs, a similar protocol was used as described earlier albeit with some adaptations (7). In short, $20 \mu \mathrm{l} \mathrm{FcXL}$ affinity matrix slurry was directly added to Pierce spin columns (ThermoFisher Scientific), followed by three washing steps with $150 \mu \mathrm{l}$ Phosphate buffer (PB, 150mM, pH7), in which for each washing step the liquid was removed by centrifugation for $1 \mathrm{~min}$ at $500 \times g$ at room temperature. After washing, the $2 \mathrm{~mL}$ tube was replaced by a $1.5 \mathrm{~mL}$ tube. The affinity matrix was resuspended in $150 \mu \mathrm{PB}$ for the unbound and full plasma fraction and in $40 \mu \mathrm{l}$ PB with a $1 \%$ blocking buffer background (Bio-Rad, The Netherlands) for the bound fractions. Subsequently $50 \mu \mathrm{l}$ of the unbound fraction, $20 \mu$ l of full plasma or the whole bound fraction $(360 \mu \mathrm{l})$ were added to the corresponding affinity matrix. Furthermore, $1 \mu \mathrm{l}$ of a solution containing two monoclonal antibodies (mAbs) (i.e., trastuzumab and alemtuzumab) at $200 \mu \mathrm{g} / \mathrm{mL}$ each, was added as internal standard for quantification to the unbound and plasma fraction. Alternatively, $1 \mu \mathrm{l}$ of a solution containing these $\mathrm{mAbs}$ at $50 \mu \mathrm{g} / \mathrm{mL}$ was added to the bound fraction. The samples were then incubated under shaking conditions for one hour at room temperature. After incubation, the flowthrough was collected and the affinity matrix with bound IgGs was washed four times with $200 \mu \mathrm{l}$ PB. Finally, $50 \mu \mathrm{PB}$ containing $50 \mathrm{U}$ of immunoglobulin degrading enzyme (IgdE; branded FabALACTICA, Genovis AB, Lund, Sweden) was added, which selectively cleaves only IgG1s in their hinge region, before incubation on a thermal shaker at $37^{\circ} \mathrm{C}$ for at least 16 hours. After overnight incubation with lgdE, the flowthrough containing the Fab fragments generated from the bound IgG1s was collected by centrifugation for $1 \mathrm{~min}$ at $500 \times g$. Using this approach, we generated IgG1 Fab samples from full plasma, and from the bound and unbound fraction for all included donors, following affinity purification with any of the four VOCs S-protein variants.

\section{LC-MS profiling}

For the mass analysis of the intact released Fabs an LC-MS and data processing approach was used as described by Bondt et al. (7). In short, the collected intact Fab proteins were separated by using a Thermo Scientific Vanquish Flex UHPLC instrument, equipped with a 1 × $150 \mathrm{~mm}$ MAbPac Reversed Phase HPLC Column. Both the column preheater and the analytical column chamber were heated to $80^{\circ} \mathrm{C}$ during chromatographic separation. The LC was directly coupled to an Orbitrap Exploris 480 mass spectrometer with BioPharma option (Thermo Fisher Scientific, Bremen, Germany). The Fab samples were separated over a 62 min gradient at a $150 \mu \mathrm{l} / \mathrm{min}$ flow rate. Gradient elution was achieved using two mobile phases, $\mathrm{A}(0.1 \% \mathrm{HCOOH}$ in Milli-Q water) and $\mathrm{B}(0.1 \%$ $\mathrm{HCOOH}$ in $\mathrm{CH} 3 \mathrm{CN}$ ) at a starting mixture of $90 \% \mathrm{~A}$ and $10 \% \mathrm{~B}$, ramping up from $10 \%$ to $25 \%$ over $1 \mathrm{~min}$, from $25 \%$ to $40 \%$ over $54 \mathrm{~min}$, and from $40 \%$ to $95 \%$ over 1 min. MS data were collected with the instrument operating in intact protein and low-pressure mode. 
Spray voltage was set at $3.5 \mathrm{kV}$ from minute 2 to minute 50 to prevent salts in the sample from entering the MS, ion transfer tube temperature was set at $350^{\circ} \mathrm{C}$, vaporizer temperature at $100{ }^{\circ} \mathrm{C}$, sheath gas flow at 15 arb.units, auxiliary gas flow at 5 arb.units, and source-induced dissociation (SID) was set at $15 \mathrm{~V}$. Spectra were recorded with a resolution setting of 7500 (@m/z 200) in MS1 allowing improved detection of charge distributions of large proteins (>30kDa) (9). Scans were acquired in the range of $500-4000 \mathrm{~m} / \mathrm{z}$ using an automated gain control (AGC) target of $300 \%$ and a maximum injection time set to $50 \mathrm{~ms}$. For each scan 5 uscans were recorded.

\section{Data analysis}

The retention times and masses of each of the Fab molecules were retrieved from the generated RAW files using BioPharmaFinder 3.2 (Thermo Scientific). Deconvolution was performed using the ReSpect algorithm between 5 and 57 minutes using $0.1 \mathrm{~min}$ sliding windows with $25 \%$ offset and a merge tolerance of $30 \mathrm{ppm}$, and noise rejection set at $95 \%$. The output range was set at 10,000-100,000 Da with a target mass of $48,000 \mathrm{Da}$ and mass tolerance of $30 \mathrm{ppm}$. Charge states between 10 and 60 were included, and the Intact Protein Peak model was selected. Further data analysis was performed using in-house scripts using Python 3.8.3 (with libraries: Pandas 1.0.5, Numpy 1.18.5, Scipy 1.5.0, matplotlib 3.2 .2 and seaborn 0.11.0). Masses of the BioPharmaFinder identifications (components) were recalculated using an intensity weighted mean, considering only the most intense peaks comprising $90 \%$ of the total intensity. Furthermore, using the data of the two spiked-in recombinant mAbs (i.e., trastusumab and alemtuzumab) the intensity could be normalized, and each Fab clonal signal quantified to a concentration in $\mu \mathrm{g} / \mathrm{ml}$.

Components between 45,000 and 53,000 kDa with the most intense charge state above $\mathrm{m} / \mathrm{z} 1,000$ and BPF score $>=40$ were considered likely Fab fragments of IgG1 clones. Clones within a 1.4 Da mass and $0.8 \mathrm{~min}$ retention-time window were considered identical.

\section{Binding and Neutralization assays}

\section{Protein coupling to Luminex beads}

To measure the binding of $\lg G$ to the S-proteins of different VOCs, we covalently coupled pre-fusion stabilized S-proteins to Luminex Magplex beads using a two-step carbodiimide reaction as previously described (10). In short, Luminex Magplex beads (Luminex) were washed with $100 \mathrm{mM}$ monobasic sodium phosphate $\mathrm{pH} 6.2$ and activated by addition of Sulfo-N-Hydroxysulfosuccinimide (Thermo Fisher Scientific) and 1-Ethyl-3-(3-dimethylaminopropyl) carbodiimide (Thermo Fisher Scientific) and incubated for $30 \mathrm{~min}$ on a rotator at room temperature. After washing the activated beads three times with $50 \mathrm{mM}$ MES pH 5.0, the S-proteins were added in ratio of 75 $\mu \mathrm{g}$ protein to 12.5 million beads and incubated for three hours on a rotator at room temperature. To block the beads for aspecific binding, we incubated the beads for 30 minutes with PBS containing $2 \%$ BSA, $3 \%$ fetal calf serum and $0.02 \%$ Tween-20 at $\mathrm{pH} 7.0$. Finally, the beads were washed and stored at $4^{\circ} \mathrm{C}$ in PBS containing $0.05 \%$ sodium azide $(11,12)$.

\section{Luminex assays}

Optimization experiments revealed the optimal concentration of the plasma for studying the humoral response following SARS-CoV-2 infection to be 10.000-fold dilution. As previously described $(10,11)$, $50 \mu \mathrm{L}$ of a bead mixture containing all different Sproteins in a concentration of 20 beads per $\mu \mathrm{L}$ were added to $50 \mu \mathrm{L}$ of diluted plasma and incubated overnight on a rotator at $4^{\circ} \mathrm{C}$. The next day, plates were washed with TBS containing $0.05 \%$ Tween-20 (TBST) and resuspended in $50 \mu \mathrm{L}$ of Goat-anti-human IgG-PE (Southern Biotech). After 2 hours of incubation on a rotator at room temperature, the beads were washed with TBST and resuspended in $70 \mu \mathrm{L}$ Magpix drive fluid (Luminex). Read-out of the plates was performed on a Magpix (Luminex). The binding of antibodies is expressed as the Median Fluorescence Intensity (MFI) of approximately 50 to 100 beads per well. MFI values are corrected for background signals by subtracting the MFI of wells containing only buffer and beads. 
Research Article

\section{Pseudo-virus construction}

The WT, Alpha, Beta and Gamma pseudovirus S constructs were ordered as gBlock gene fragments (Integrated DNA Technologies) and cloned using Sacl and Apal in the pCR3 SARS-CoV-2-S $\triangle 19$ expression plasmid (13) using Gibson Assembly (ThermoFisher). All constructs were verified by Sanger sequencing. Pseudo-viruses were produced by co-transfecting the SARS-CoV-2-S expression plasmid with the pHIV1NL43 $\triangle$ Env-NanoLuc reporter virus plasmid in HEK293T cells (ATCC, CRL-11268), as previously described (13). Cell supernatant containing the pseudo-virus was harvested 48 hours post transfection and stored at $-80^{\circ} \mathrm{C}$ until further use.

\section{Pseudo-virus neutralization assays}

Neutralization activity was tested using a pseudo-virus neutralization assay, as previously described (4). Shortly, HEK293T/ACE2 cells, kindly provided by Dr. Paul Bieniasz (13), were seeded at a density of 20,000 cells/well in a 96 -well plate coated with $50 \mu \mathrm{g} / \mathrm{mL}$ polyL-lysine one day prior to the start of the neutralization assay. NAbs (1-50 $\mu \mathrm{g} / \mathrm{mL})$ or heat-inactivated plasma samples (1:100 dilution) were serially diluted in cell culture medium (DMEM (Gibco), supplemented with $10 \%$ FBS, penicillin (100 U/mL), streptomycin (100 $\mu \mathrm{g} / \mathrm{mL}$ ) and GlutaMax (Gibco)), mixed in a $1: 1$ ratio with pseudo-virus and incubated for 1 hour at $37^{\circ} \mathrm{C}$. Subsequently, these mixtures were added to the cells in a $1: 1$ ratio and incubated for 48 hours at $37^{\circ} \mathrm{C}$, followed by a PBS wash and lysis buffer to measure the luciferase activity in cell lysates using the Nano-Glo Luciferase Assay System (Promega) and GloMax system (Turner BioSystems). Relative luminescence units (RLU) were normalized to the positive control wells where cells were infected with pseudo-virus in the absence of NAbs or plasma. The inhibitory concentration (IC50) and neutralization titers (ID50) were determined as the NAb concentration and plasmadilution at which infectivity was inhibited by $50 \%$, respectively, using a non-linear regression curve fit (GraphPad Prism software version 8.3). Samples with ID50 titers < 100 were defined as having undetectable neutralization.
IgG1 responses following SARS-CoV-2 infection

\section{Results}

\section{IgG1 clonal profiling}

The primary aim of this study was to assess the clonal diversity of SARS-CoV-2 specific IgG1 antibodies in plasma of individual donors and their cross-reactivity against the different S-protein variants representing VOCs. We analyzed the plasma of eight donors who had experienced an infection with the WT or one of the three VOCs, namely Alpha, Beta and Gamma (Figure 1A, Supplemental Table 1). All plasma samples were collected 3-6 weeks after the start of symptoms. In total we recorded 40 different plasma lgG1 repertoires using LC-MS, of which 8 were full plasma repertoires (one per donor) and $8 * 4$ were obtained from the $S$ protein variant directed sub-pools of $\operatorname{lgG} 1 \mathrm{~s}$ (Figure 1B). Across all donors the number of detected $\lg G 1$ clones in full plasma as defined by LC-MS varied between $247-517$, with the top 25 clones representing about $40-70 \%$ of the total IgG1 concentration (Supplementary Table 2). Focusing next on the Fabs retrieved by the immune-affinity pull-downs, it became noticeable that their total concentration was quite variable, with some donors producing anti S-protein IgG1s just up to a concentration of a few $\mathrm{ng} / \mathrm{mL}$ (e.g., donor 002 and 307), whereas others produced up to > $20 \mu \mathrm{g} / \mathrm{mL}$ of anti S-protein IgG1s (e.g., donor 003 and 303). This implies that in the former two donors the anti S-protein IgG1s make up less than $1 \%$ of the full plasma IgG1 repertoire, whereas in the latter case this is more in the range of $5-10 \%$. The number of unique clones identified in all repertoires is provided in Supplementary Table 2 and reveals that the total numbers align well with the total concentrations.

Except donor 303, the highest number and the highest concentration of anti S-protein IgG1s was observed, as expected, versus the S-protein originating from the VOC causing the infection. Somewhat surprising, donor 303 was infected with the Alpha variant but displayed a substantially higher quantity of anti Sprotein lgG1s in the WT pull-down when compared to the pull-down with the Alpha variant S-protein (Supplementary Figure 1), despite the high similarity between Alpha and WT $(4,14)$. 

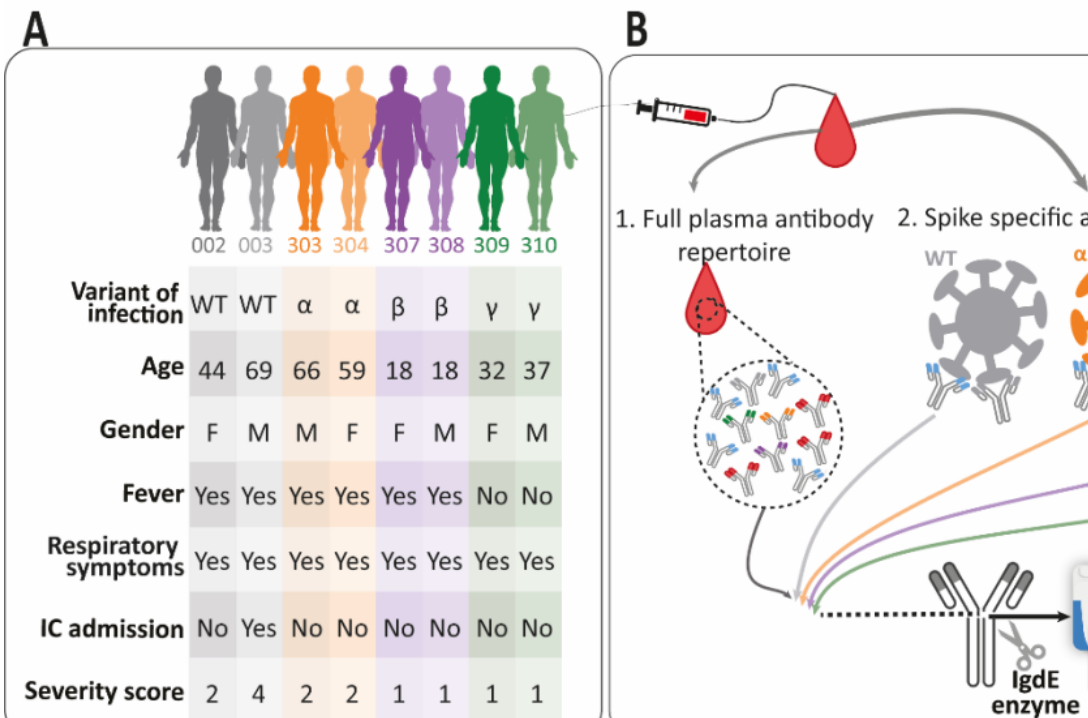

1. Full plasma antibody 2 . Spike specific antibody repertoire

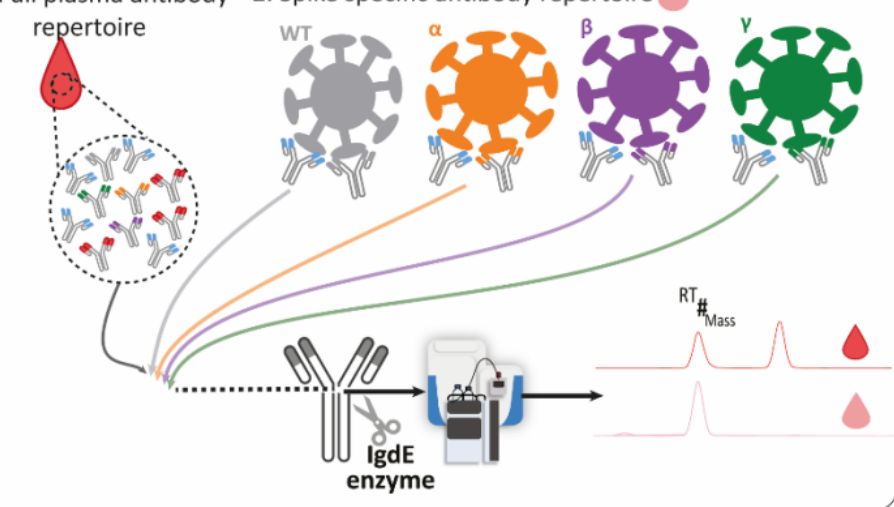

Figure 1| Donor Characteristics and Monitoring of individual full IgG1 and S-protein antigen directed IgG1 profiles. A. Overview of the donors, who had experienced an infection by the named VOCs. VOCs are color-coded with WT (gray), Alpha (orange), Beta (purple) and Gamma (green). The table also lists age, gender, and disease state following the World Health Care (WHO) severity score. B. For each plasma sample taken we analyzed the full plasma lgG1 antibody repertoire as well as the antigen directed IgG1 clones to the four different VOCs S-proteins. The experimental approach involves the IgG capturing from full plasma as well as the S-protein specific immune-capturing. Fab fragments of the IgG1s were generated by enzymatic cleavage and subsequently subjected to intact-protein LC-MS analysis. Clonal repertoires could be profiled qualitatively, whereby each identified clone can be characterized by its unique accurate mass (in Dalton) and retention time (RT in minutes). By spiking in known quantities of two recombinant $m A$ bs each plasma lgG1 clone could be quantified. The different S-protein specific Fab repertoires and the full plasma Fab repertoires were then compared, both intra- and inter-donors.

We next evaluated the diversity in these $\lg \mathrm{G} 1$ repertoires intra- and inter-donors. Every unique lgG1 clone, annotated as ${ }^{\mathrm{RT}}$ \#mass, $_{\text {mas }}$ distinguished by its accurate mass in Dalton and LC retention time (RT) in minutes. Adopting similar analysis tools as introduced earlier (7), we cross-correlated the full plasma lgG1 repertoires, using the concentration of each unique identifier ${ }^{\mathrm{RT}} \#_{\text {mass, }}$ whereby two identical profiles would provide a correlation of $100 \%$ (colored dark red in Figure 2) and a weak correlation would be just a few $\%$ (colored white in Figure 2). The observation that there is nearly no overlap in IgG1 repertoires in between donors is fully in line with our earlier data; each person's plasma lgG1 repertoire is unique and no clone is detected in more than one donor (7).

Next, we cross-correlated the four affinity pulled-down repertoires for a single donor and compared thatwith the full plasma lgG1 repertoire of that given donor. Several interesting observations can be extracted from this data. For some donors the S-protein directed IgG1 repertoires are within that donor quite alike (e.g. donors 003, 303, 304 and 310), no matter which Sprotein had been used as affinity handle. For donor 003 these four S-protein directed IgG1 repertoires also cross-correlate quite well with the total IgG1 repertoire, indicating that several abundant clones in the plasma of this donor are directed against the S-protein of SARS-CoV-2 (Figure 2). In contrast, with donor 002 the correlation among the four pull-downs is quite high, but the correlation with the full plasma lgG1 repertoire of that donor is close to zero, indicating that the majority of abundant clones found in the plasma of this donor are not directed against any of the four Sproteins.

Overall, we observe that in these repertoires quite a few clones are present that cross-react with other Sprotein variants, although other clones bind more restrictively to only one (or a few) variants, as we will discuss in further detail below. 
Research Article

IgG1 responses following SARS-CoV-2 infection

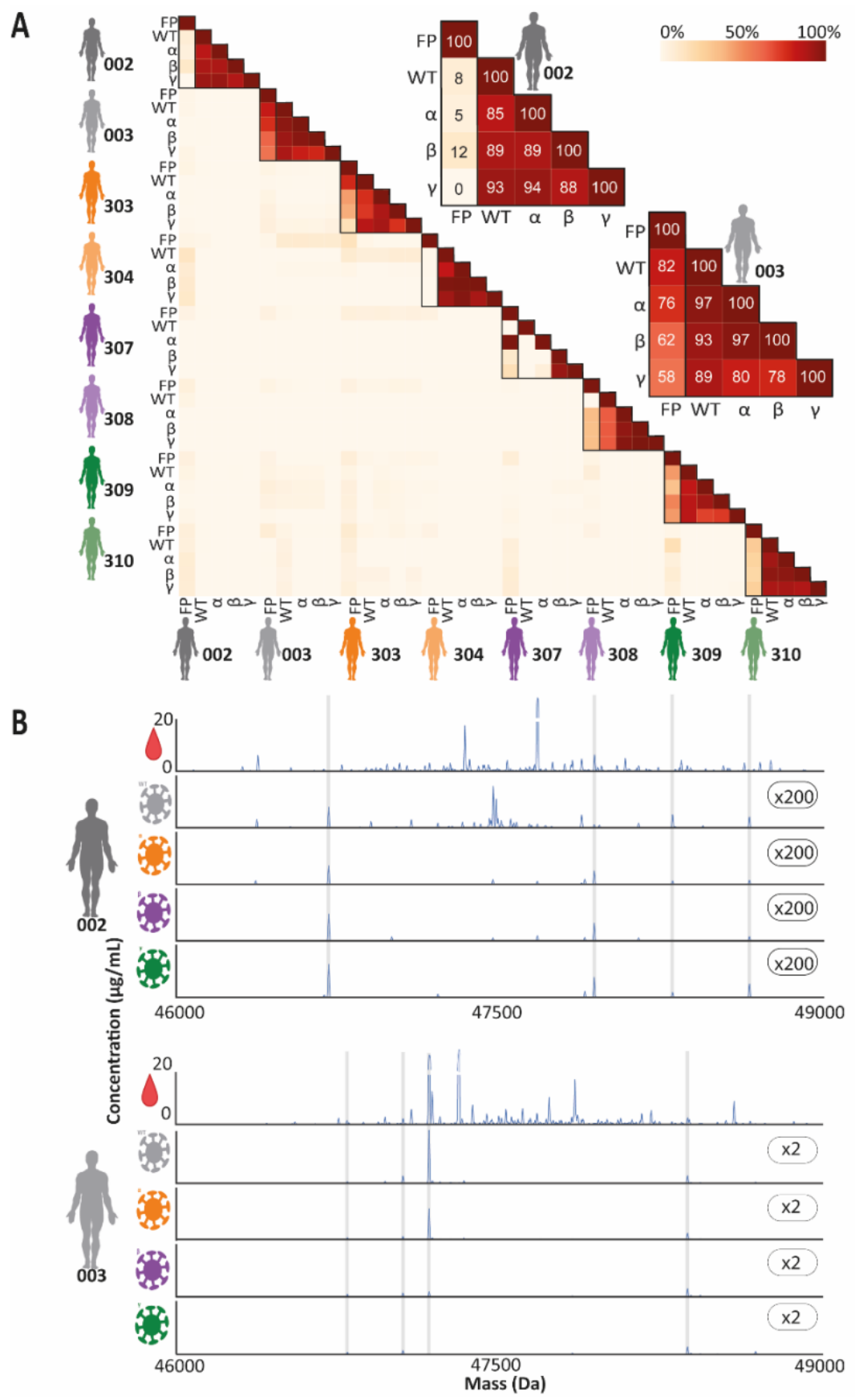

Figure 2| S-protein specific IgG1 repertoires are polyclonal and unique per donor, whereas substantial crossreactivity is observed when antigen-binding IgG1 pools of a given donor are enriched by either one of the four VOCs S-protein trimers. A. Quantitative overlap of IgG1 repertoires illustrated by a heatmap, depicting the degree of overlap between all detected IgG1 repertoires, i.e. from all donors, extracted from either the full plasma (FP) or after pulldowns with each of the VOCs S-protein of all variants studied (WT, Alpha, Beta and Gamma). The quantitative overlap

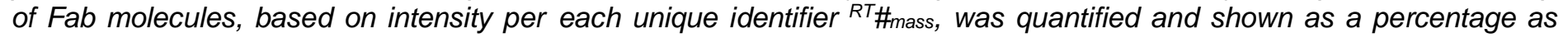
indicated by the color bar. The zoomed-in panels for donors 002 and 003 highlight the substantial overlap for donor 003 between the S-protein specific Fab profiles, and the full plasma IgG1 profile. B. Deconvoluted full Fab mass profiles as obtained for donor 002 and 003, from the S-protein specific Fab profiles (WT, Alpha, Beta and Gamma) and the full plasma Fab profile (top). Each peak represents a unique Fab at its detected mass and plasma concentration. The number on the right of each S-protein specific profile, indicated the $y$-axis multiplier compared to the $y$-axis used for the full plasma profiles. Supplementary Figure 2-7 depict the full Fab mass profiles of all the other six donors. 


\section{Several antigen-directed IgG1 clones display cross-reactivity versus the VOCs S-protein variants}

Next, we focused on donor 003, who had developed most severe Covid-19, as indicated by the WHO severity score, and had been hospitalized after being infected with the WT-variant. For this donor, we were able to pull-down between 68 and 192 distinctive lgG1 clones with each of the S-protein variants tested, clearly revealing a quite broad polyclonal response (Supplementary Table 2). In Figure $3 \mathrm{~A}$ and $3 \mathrm{~B}$ an overview of the ten most abundant S-protein directed $\lg \mathrm{G} 1$ clones is given (with their identifier ${ }^{\mathrm{RT}} \#_{\text {mass }}$ ), depicting the quantity of each of the clones when affinity-enriched by each of the four S-protein variants. Clearly, the clone annotated as ${ }^{19.2} 64$ 47,172.5 is the most abundant clone pulled down with the WT S-protein. This clone is also very abundant in the pull-down with the Alpha S-protein, but much less so following the pull-down with the Beta S-protein and is almost not captured when the Gamma S-protein is used. In contrast, other clones display wide and somewhat equal cross-reactivity against all four variants, such as 20.3142 47,792.9 and ${ }^{18.3} 248$ 48,373.7. Yet another IgG1 clone is only pulled down with the WT and Gamma variant; ${ }^{22.1} 207$ 48,689.7. In Figure $3 \mathrm{C}$ a radar plot provides further insight in how each clone behaves in having distinctive affinity for the different $\mathrm{S}$-protein variants. This data is compared with the radar plot obtained by summing all IgG1s having affinity for the S-proteins, as the latter is typically measured when total titers are assessed in donors. From this analysis it is directly clear that not every clone follows the same trend as observed for the total antigen directed IgG1 titer. Although we focused in this section on the responses of donor 003 , alike observations were made for other donors, with generally each clone exhibiting its own pattern of reactivity against the four tested Sproteins.

Donor 303 is somewhat remarkable, in the sense that it harbors one clone, $20.144948,655.2$, with a more than 10 times higher affinity for the WT S-protein variant compared to Alpha, although the latter was the variant of infection. Again, there are also clones in this donor that show the same binding towards all S-protein variants. Another interesting trend for the clones found in this donor is, that the clones that appear to bind to the Beta S-protein variant are barely showing binding to the Gamma S-protein variant and vice versa (Supplementary figure 1).

Clonal repertoire properties and correlation with complementary binding and neutralization assays More standard approaches to evaluate antibodydependent immune responses of individual donors use either Luminex bead-based binding assays or virus neutralization assays $(8,10,15,16)$. Thus, to corroborate our findings, we next performed such complementary neutralization and binding assays (Figure 4). Neutralization was measured as ID50 (plasma dilution that inhibits $50 \%$ of the infectivity) and the S-protein specific IgG binding was measured using a fluorescently labeled secondary antibody to detect $S$ protein binding $\lg G$, resulting in a mean fluorescence intensity (MFI) $(8,14)$. To compare these results with the LC-MS based clonal profiling, we calculated the sum of the concentration of all detected IgG1 clones that were found to bind to the VOC specific S-protein variants in each donor (Figure 4B). Of note, while the LC-MS based clonal profiling described above is IgG1 specific, the binding assay assess the full repertoire of IgGs (IgG1, IgG2, IgG3 and IgG4), and the neutralization assay the full repertoire of $\operatorname{lgs}(\lg G, \lg A$, $\lg M$ ) thus the results may be different, albeit that $\lg G 1$ is generally the most abundant subclass in plasma.

For donors 002, 003 and 304 the response to the VOC of infection dominates, as a priori expected, the radar plots in Figure 4A. However, we also observe clear divergences. For example, donor 303 shows the highest neutralization and IgG binding for the variant of infection (Alpha), but the anti-S protein IgG1 concentration is much higher with the WT variant. For donor 307 the neutralization and specific lgG1 is much higher for the Gamma variant then for the Beta variant that infected this donor. Overall, there is quite some inconsistency between the three assays as seen in high total lgG binding vs low IgG1 specific binding (e.g. donor 304), or low neutralization vs high $\lg G(1)$ binding 
(e.g. donor 308). However, also all this data reveals that Igs from a given donor, whereby infection as caused by a certain VOC, do cross-react quite well with viruses from other VOCs, thus widespread crossreactivity is corroborated by all three assays.

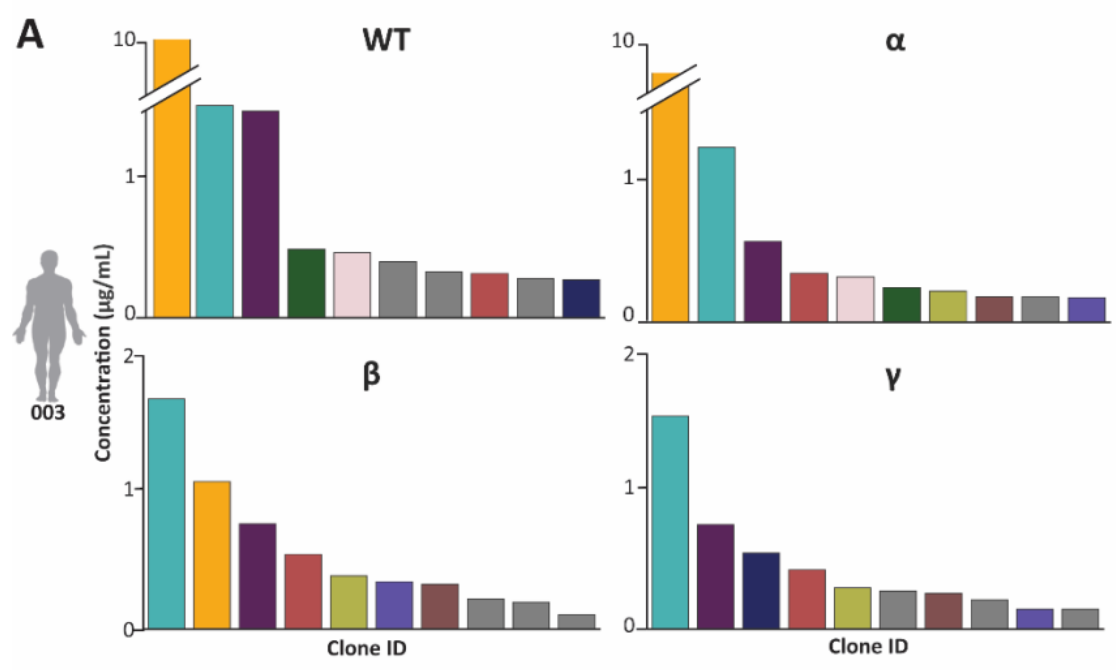

B

C

\begin{tabular}{|c|c|c|c|c|}
\hline $\begin{array}{l}\text { Clone ID } \\
\left(\stackrel{\text { RT (min) }}{\#}{ }_{\text {Mass (Da) }}\right)\end{array}$ & $\begin{array}{c}\text { WT } \\
(\mu \mathrm{g} / \mathrm{mL})\end{array}$ & $\begin{array}{c}\alpha \\
(\mu \mathrm{g} / \mathrm{mL})\end{array}$ & $\begin{array}{c}\beta \\
(\mu \mathrm{g} / \mathrm{mL})\end{array}$ & $\begin{array}{c}V \\
(\mu \mathrm{g} / \mathrm{mL})\end{array}$ \\
\hline $\begin{array}{lll}19.1 & 64 & 47,172.5\end{array}$ & 11.5 & 6.2 & 1.1 & 0.1 \\
\hline${ }^{18.3} 248 \quad 48,373.7$ & 1.5 & 1.3 & 1.5 & 1.7 \\
\hline $23.34947,051.1$ & 1.5 & 0.6 & 0.8 & 0.8 \\
\hline${ }^{18.9} 109 \quad 47,335.0$ & 0.5 & 0.3 & 0.1 & N.D. \\
\hline${ }^{17.6} 62{ }_{47,188.7}$ & 0.5 & 0.3 & 0.1 & N.D. \\
\hline${ }^{20.3} 142 \quad 46,792.9$ & 0.3 & 0.4 & 0.5 & 0.4 \\
\hline $22.120748,689.7$ & 0.3 & N.D. & N.D. & 0.6 \\
\hline $19.4143 \quad 46,792.4$ & 0.3 & 0.2 & 0.4 & 0.3 \\
\hline${ }^{18.5} 243 \quad 48,389.2$ & 0.2 & 0.2 & 0.3 & 0.3 \\
\hline $\begin{array}{lll}19.4 & 253 & 48,432.7\end{array}$ & 0.2 & 0.2 & 0.3 & 0.3 \\
\hline
\end{tabular}

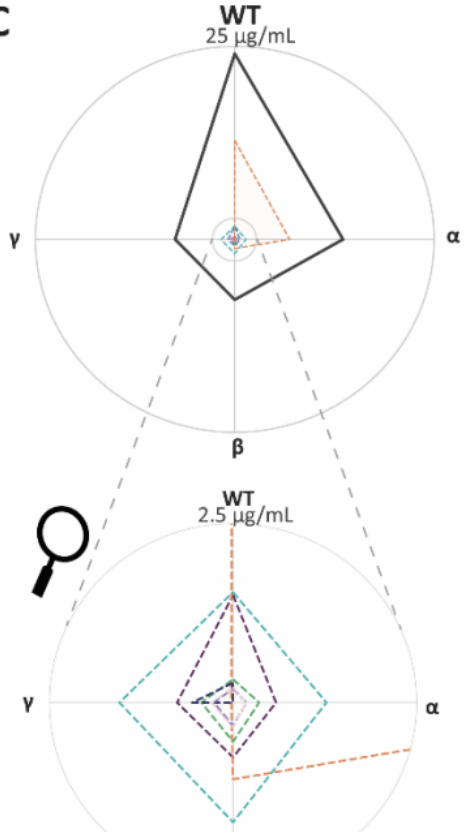

Figure 3| Within a single donor, antigen directed IgG1 clones display distinctive cross-reactivity versus the VOCs S-protein variants. A. Quantitative comparison of the ten most abundant IgG1 Fab clones, affinity-enriched from the plasma of donor 003, with each of the four S-protein variants. Each bar represents one of the 10 most abundant clones with the height indicating the concentration in $\mu \mathrm{g} / \mathrm{ml}$, using the same colors for the clones corresponding to panel $B$. Each clone that was not in the total top 10 but was in the top 10 for that specific VOC is colored grey. B. Top 10 most abundant clones with their relative abundance after the pull-down with each of the four VOCs S-protein variants. The colored bars in $(A)$ and the dotted lines in the radar plot in $(C)$ are corresponding to the clones in the table B. C. Radar plot with on each edge the data for one of the S-protein variants. These plots depict the difference in binding of specific clones against the VOCs Sprotein variants. The thick solid black line representing the sum of all enriched lgG1 clones. Color coding is identical as used in $(A)$ and $(B)$. 


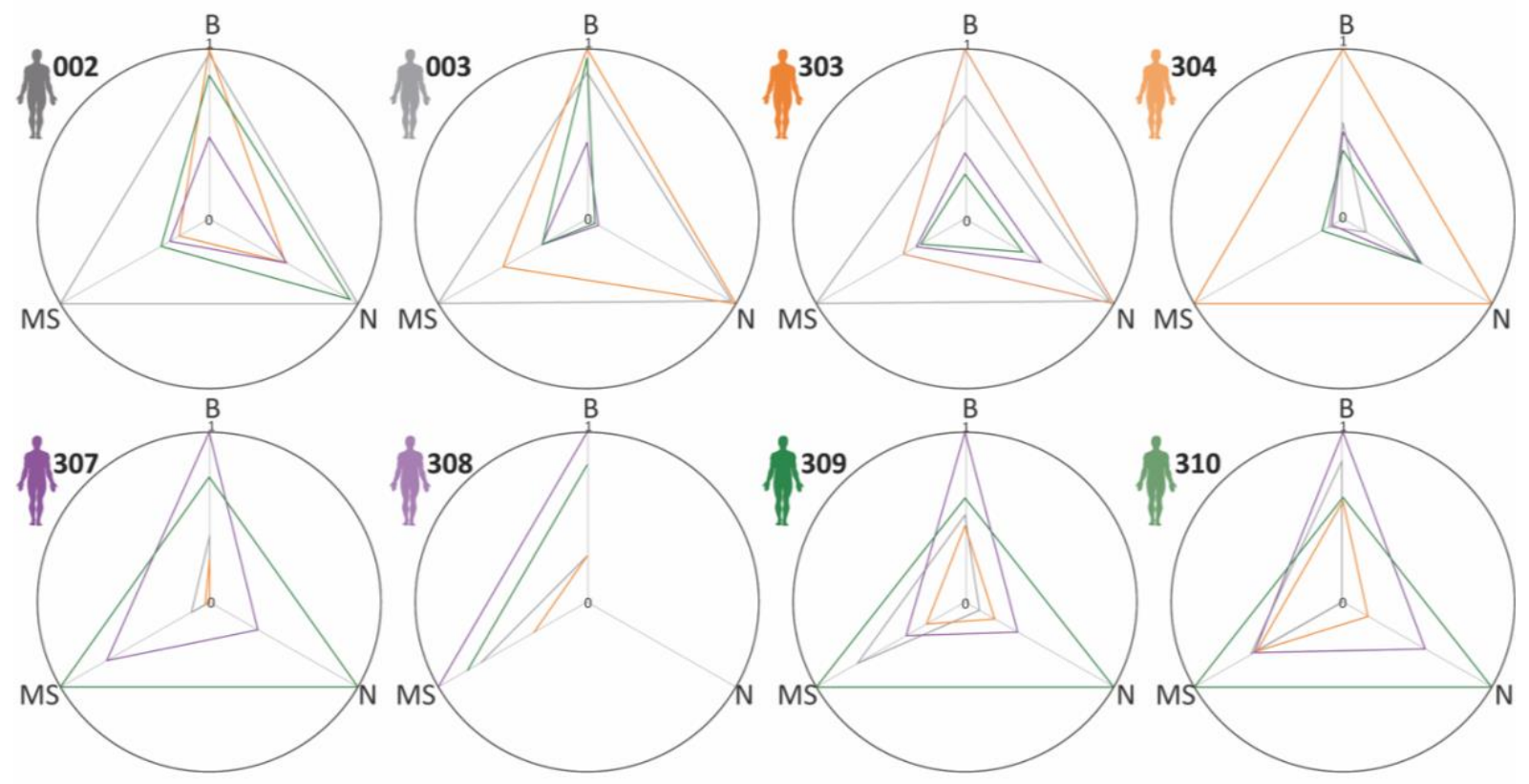

\begin{tabular}{|c|c|c|c|c|c|c|c|c|c|c|c|c|}
\hline & \multicolumn{4}{|c|}{ Neutralization $\left(\mathrm{ID}_{50}\right)$} & \multicolumn{4}{|c|}{ Spike specific IgG binding (MFI) } & \multicolumn{4}{|c|}{ Spike specific lgG1 binding $(\mu \mathrm{g} / \mathrm{mL})$} \\
\hline & WT & $\alpha$ & $\beta$ & $y$ & WT & $\alpha$ & $\beta$ & $y$ & WT & $\alpha$ & $\beta$ & $\gamma$ \\
\hline 002 & 1552 & 796 & 807 & 1469 & 1800 & 1856 & 892 & 1569 & 0.6 & 0.2 & 0.1 & 0.2 \\
\hline 003 & 22262 & 22907 & 1744 & 1212 & 7987 & 9274 & 4170 & 8775 & 21.7 & 12.3 & 7.0 & 6.7 \\
\hline 303 & 2169 & 34626 & 11719 & 8943 & 18852 & 26068 & 10121 & 6862 & 31.6 & 14.6 & 10.1 & 9.5 \\
\hline 304 & 169 & 1080 & 570 & 555 & 1334 & 2352 & 1202 & 947 & 0.6 & 0.7 & 0.6 & 0.9 \\
\hline 307 & $<100$ & $<100$ & 319 & 977 & 1029 & 676 & 2640 & 1944 & $<0.1$ & $<0.1$ & $<0.1$ & $<0.1$ \\
\hline 308 & $<100$ & $<100$ & $<100$ & $<100$ & 59 & 69 & 218 & 176 & 0.3 & 0.1 & 0.3 & 0.3 \\
\hline 309 & 307 & 630 & 1120 & 3163 & 3069 & 2685 & 5982 & 3673 & 2.3 & 0.8 & 1.2 & 3.1 \\
\hline 310 & $<100$ & 283 & 927 & 1678 & 3458 & 2473 & 4168 & 2574 & 0.3 & 0.3 & 0.3 & 0.5 \\
\hline
\end{tabular}

Figure 4| Properties of antigen specific $\operatorname{lgG}(1)$ repertoires and correlation between different binding and neutralization assays. A. each radar plot represents data for a single donor, with axis representing data of the neutralization assay (N), S-protein specific total IgG binding assay (B) and S-protein specific lgG1 binding by LC-MS (MS). Per axis and per donor, the values are normalized based on the highest value observed for that axis for that specific donor, giving a value 1 with the other values displaying the proportion of this highest base value, i.e. in between 0 and 1. Each line represents data against a particular S-protein (shown with the color of the line) for that specific donor. B. Values behind the radar plots in $A$. ID $D_{50}$ determined in the neutralization assays (left), and amount of S-protein specific IgG binding observed by the fluorescence assay (middle) and the LC-MS based clonal profiling (right). In the latter assay S-protein specific binding is calculated by summing up the concentrations of the S-protein specific clones for that specific donor. The colors represent the high (in red) to low (light yellow) values determined per individual assay 


\section{Discussion and Conclusion}

The S-protein of SARS-CoV-2 is prone to mutations, stirring up questions regarding the protection of antibodies directed against one variant towards the other variants. Most existing assays to measure antibody cross-reactivity against different S-protein variants lack the discriminatory power to provide insights at the level of individual unique antibody clones. Here we developed a direct MS-based approach enabling the analysis of the polyclonal response of individuals producing SARS-CoV-2 Sprotein targeting lgG1 clones. Our data reveal that every donor shows a unique plasma IgG1 repertoire (7), but also a unique anti S-protein IgG1 repertoire, with each clone showing a distinct pattern of crossreactivity versus different variants.

That each clone shows a unique binding pattern can be caused by the unique binding epitopes of the IgG clones in their interactions with the S-protein, leading to that some clones are more affected by specific mutations $(17,18)$. In addition, factors such as avidity and (anti-) cooperativity are known to also influence binding between full-length IgG1 and the S-protein (19, 20). In any case, these binding differences between antibody clones are an important feature to consider when selecting antibodies for further development into biotherapeutics. Here we show that not always the most abundant antibody exhibits the best crossreactivity against all other S-protein variants.

Our data show that IgG binding does not always correlate with neutralization what may suggest alternative functions for these S-protein binding Igs. Moreover, there is evidence from the literature that antibodies against SARS-CoV-2 with low IgG fucosylation result in increased macrophage activation, thereby introducing the antibody dependent cellular cytotoxicity (ADCC) and phagocytose and with that highlighting the broad functionality of antibodies also during and after SARS-CoV-2 infection $(21,22)$. Besides, whether a specific antibody against SARSCoV-2 will neutralize or not is likely also epitope dependent $(17,23)$. The difference we observe for some donors between total IgG binding and IgG1 binding to the S-protein furthermore suggests donorspecific immune responses which could be dominated by $\lg G$ subclasses other than $\lg G 1$. Next to $\lg G 1$, IgG3 has been reported to be broadly involved during SARS-CoV-2 infection (24-26). These differences observed between donors, again, highlight the unique donor-specific humoral immune responses towards SARS-CoV-2.

Although here we assessed the polyclonal IgG1 responses in donors suffering a SARS-CoV-2 infection, our direct LC-MS method may equally well be exploited to monitor responses induced by infections with other pathogens like viruses and bacteria, and even certain diseases such as cancer and rheumatoid arthritis.

Moreover, although this study was restricted to the VOCs known at the time of conducting the experiments, it can be extended to other and new VOCs. Our data are in agreement with the finding that humans may (have) acquire(d) pre-existing crossreactive humoral immunity, mostly against the $\mathrm{S} 2$ subunit of the S-protein, as this part is mostly conserved between the different S-protein variants of SARS-CoV-2 and among the sequences of related group B coronaviruses $(11,17,27,28)$. It is also the S1 subunit that is shown to be mostly mutated in the Delta and Omicron VOCs, suggesting that the preexisting cross-reactivity against the $\mathrm{S} 2$ subunit is not that much affected. This, together with the unique Sprotein specific response we observed for each donor, could explain why some individuals' repertoires better protect against re-infection with another VOC than others.

In conclusion, by assessing IgG1 repertoires following infection we observe a widespread polyclonal lgG1 response in SARS-CoV-2 infected people that are unique for each person. Each clone exhibited a distinct pattern of cross-reactivity versus SARS-CoV-2 Sprotein variants. Furthermore, the clonal repertoire analysis did not per se correlate with in vitro neutralization assays, highlighting the need for a variety of assays to judge the full scale of antiviral 
Research Article

fitness of a patient sample. The knowledge that every clone shows a different SARS-CoV-2 bindings pattern, is important to consider when developing new biotherapeutics or novel vaccination strategies. And while here shown for SARS-CoV-2, the here described approach can be further utilized to have a detailed look into the antigen-specific antibody response in a wide variety of diseases.

\section{Author Contributions}

D.M.H.v.R, A.B., M.J.v.G., and A.J.R.H. came up with the concept and designed the research; K.v.d.S., D.E, C.R., R.W.S., and G.J.d.B set up the COSCA study and recruited the study participants; D.M.H.v.R., A.B, K.v.d.S., T.G.C. and M.P. performed experiments; D.M.H.v.R., A.B., M.H., K.v.d.S. and A.J.R.H analyzed the data; and D.M.H.v.R., A.B., and A.J.R.H. wrote the paper, which was edited by all co-authors.

\section{Acknowledgements}

This research received funding through the Dutch Research Council (NWO) funding the Netherlands Proteomics Centre through the X-omics Road Map program (project 184.034.019) and Gravitation Subgrant 00022 from the Institute for Chemical Immunology (D.M.H.v.R. and A.B.). A.J.R.H. acknowledges support from the Netherlands Organization for Scientific Research (NWO) through the Spinoza Award SPI.2017.028 to A.J.R.H.. R.W.S. acknowledges support from the Netherlands Organization for Scientific Research (NWO) through a Vici grant 91818627 . The authors also acknowledge support from the Bill \& Melinda Gates Foundation through grant INV-024617 (M.J.v.G.). M.J.v.G. is a recipient of an Amsterdam UMC AMC Fellowship.

\section{References}

1. W. T. Harvey et al., SARS-CoV-2 variants, spike mutations and immune escape. Nat Rev Microbiol 19, 409-424 (2021).

2. WHO (2022) Tracking SARS-CoV-2 variants.
IgG1 responses following SARS-CoV-2 infection

3. I. Lazarevic, V. Pravica, D. Miljanovic, M. Cupic, Immune Evasion of SARS-CoV-2 Emerging Variants: What Have We Learnt So Far? Viruses 13 (2021).

4. T. G. Caniels et al., Emerging SARS-CoV-2 variants of concern evade humoral immune responses from infection and vaccination. Sci Adv 7, eabj5365 (2021).

5. R. J. Cox, K. A. Brokstad, Not just antibodies: B cells and T cells mediate immunity to COVID-19. Nat Rev Immunol 20, 581-582 (2020).

6. G. Georgiou et al., The promise and challenge of high-throughput sequencing of the antibody repertoire. Nat Biotechnol 32, 158-168 (2014).

7. A. Bondt et al., Human plasma IgG1 repertoires are simple, unique, and dynamic. Cell Syst 12, 11311143.e1135 (2021).

8. P. J. M. Brouwer et al., Potent neutralizing antibodies from COVID-19 patients define multiple targets of vulnerability. Science 369, 643-650 (2020).

9. M. van de Waterbeemd et al., Dissecting ribosomal particles throughout the kingdoms of life using advanced hybrid mass spectrometry methods. Nat Commun 9, 2493 (2018).

10. M. W. Keuning et al., Saliva SARS-CoV-2 Antibody Prevalence in Children. Microbiol Spectr 9, e0073121 (2021).

11. M. Grobben et al., Cross-reactive antibodies after SARS-CoV-2 infection and vaccination. Elife $\mathbf{1 0}$ (2021).

12. M. J. van Gils et al., Four SARS-CoV-2 vaccines induce quantitatively different antibody responses against SARS-CoV-2 variants. medRxiv 10.1101/2021.09.27.21264163, 2021.2009.2027.21264163 (2021).

13. F. Schmidt et al., Measuring SARS-CoV-2 neutralizing antibody activity using pseudotyped and chimeric viruses. J Exp Med 217 (2020).

14. K. van der Straten et al., Mapping the antigenic diversification of SARS-CoV-2. medRxiv 10.1101/2022.01.03.21268582, 2022.2001.2003.21268582 (2022).

15. B. Ju et al., Human neutralizing antibodies elicited by SARS-CoV-2 infection. Nature 584, 115-119 (2020).

16. J. Mariën et al., Evaluating SARS-CoV-2 spike and nucleocapsid proteins as targets for antibody detection in severe and mild COVID-19 cases using 
Research Article

a Luminex bead-based assay. J Virol Methods 288, 114025 (2021).

17. W. A. Haynes et al., High-resolution epitope mapping and characterization of SARS-CoV-2 antibodies in large cohorts of subjects with COVID19. Commun Biol 4, 1317 (2021).

18. N. S. Lipman, L. R. Jackson, L. J. Trudel, F. WeisGarcia, Monoclonal versus polyclonal antibodies: distinguishing characteristics, applications, and information resources. Ilar j 46, 258-268 (2005).

19. V. Yin et al., Probing Affinity, Avidity, Anticooperativity, and Competition in Antibody and Receptor Binding to the SARS-CoV-2 Spike by Single Particle Mass Analyses. ACS Cent Sci 7, 18631873 (2021).

20. M. Yuan et al., Structural and functional ramifications of antigenic drift in recent SARS-CoV2 variants. Science 373, 818-823 (2021).

21. W. Hoepel et al., High titers and low fucosylation of early human anti-SARS-CoV-2 IgG promote inflammation by alveolar macrophages. Sci Transl Med 13 (2021).

22. $\mathrm{Y}$. $\mathrm{Yu}$ et al., Antibody-dependent cellular cytotoxicity response to SARS-CoV-2 in COVID-19
IgG1 responses following SARS-CoV-2 infection

patients. Signal Transduct Target Ther 6, 346 (2021).

23. Y. Zhou et al., Enhancement versus neutralization by SARS-CoV-2 antibodies from a convalescent donor associates with distinct epitopes on the RBD. Cell Rep 34, 108699 (2021).

24. A. D. Moura et al., Assessment of avidity related to IgG subclasses in SARS-CoV-2 Brazilian infected patients. Sci Rep 11, 17642 (2021).

25. S. Kallolimath et al., Highly active engineered IgG3 antibodies against SARS-CoV-2. Proc Natl Acad Sci U S A 118 (2021).

26. J. K. lies et al., Determination of IgG1 and IgG3 SARS-CoV-2 spike protein and nucleocapsid binding - Who is binding who and why? medRxiv 10.1101/2021.06.17.21259077, 2021.2006.2017.21259077 (2021).

27. E. Fraley et al., Cross-reactive antibody immunity against SARS-CoV-2 in children and adults. Cell Mol Immunol 18, 1826-1828 (2021).

28. K. W. Ng et al., Preexisting and de novo humoral immunity to SARS-CoV-2 in humans. Science 370, 1339-1343 (2020). 
bioRxiv preprint doi: https://doi.org/10.1101/2022.02.24.481778; this version posted February 26, 2022. The copyright holder for this preprint (which was not certified by peer review) is the author/funder, who has granted bioRxiv a license to display the preprint in perpetuity. It is made available under aCC-BY-ND 4.0 International license.

Research Article

IgG1 responses following SARS-CoV-2 infection 